Case report

\title{
A practical solution for coil migration and coronary artery dissection in the same patient with coronary artery fistula
}

\author{
Hasan Güngör *, Cemil Zencir, Çağdaş Akgüllü, Ufuk Eryılmaz \\ Adnan Menderes University, Department of Cardiology, Aydin, Turkey
}

\section{A R T I C L E I N F O}

\section{Article history:}

Received 22 November 2015

Received in revised form 10 December 2015

Accepted 11 December 2015

Available online 5 March 2016

\section{Keywords:}

Coil

Dissection

Fistula

Migration

\begin{abstract}
A B S T R A C T
Coronary artery fistulas (CAF) are defined as a direct communication between a coronary artery and any cardiac chamber or vessel. They are generally congenital and the majority occurs in the right coronary artery (RCA) almost always draining to the right cavities and exact incidence of CAF is unknown. The treatment options for CAF are surgery or catheter closure. Catheter closure of the CAF is now considered to be an effective and safe method alternative to surgery. We report the first case with coil migration and coronary dissection to occur simultaneously in the same patient and successful treatment of these two complications with stent implantation. (c) 2016 The Society of Cardiovascular Academy. Production and hosting by Elsevier B.V. All rights reserved. This is an open access article under the CC BY-NC-ND license (http://creativecommons.org/licenses/by-nc-nd/4.0/).
\end{abstract}

A 68-year-old man was admitted to our department with complaints of exertional dyspnea and chest pain. Coronary angiography did not show significant lesions in the epicardial coronary arteries and a fistula was observed from the proximal portion of RCA draining into the pulmonary artery (Fig. 1). For embolisation a 0.014î and 300 cm hydrophilic guidewire was moved up to the distal segment of the fistula and a microcatheter was then loaded over this and a $2 \times 25 \mathrm{~mm}$ coil (Balt, Montmorency, France) was placed. For total occlusion achievement a second $2 \times 50 \mathrm{~mm}$ coil (Balt, Montmorency, France) was placed (Fig. 2). Unfortunately soon after the insertion of the second coil, during contrast injection this coil detached and migrated to proximal portion of RCA. At the same time coronary dissection was observed in the osteal part of RCA (Fig. 3). Therefore, $4.0 \times 9 \mathrm{~mm}$ bare metal stent (Simchrome $\mathrm{CoCr}$, Simeks, Turkey) was successfully implanted at 14 atm to cover the dissected segment and migrated coil was jailed to side branch to prevent any embolization to the distal portion of RCA or aorta immediately (Fig. 4). Electrocardiogram and Troponin levels were normal and patient was discharged after a 72-hour follow-up without any complication.

The clinical symptoms of CAF depend on the severity of the left-right shunt and the coronary steal. Closure is indicated for symptomatic

\footnotetext{
* Corresponding author at: Adnan Menderes University, Faculty of Medicine, Department of Cardiology, 09100 Aydin, Turkey. Tel.: +90 5068892238 (mobile). E-mail address: drgungorhasan@yahoo.com (H. Güngör).

Peer review under responsibility of The Society of Cardiovascular Academy.
}

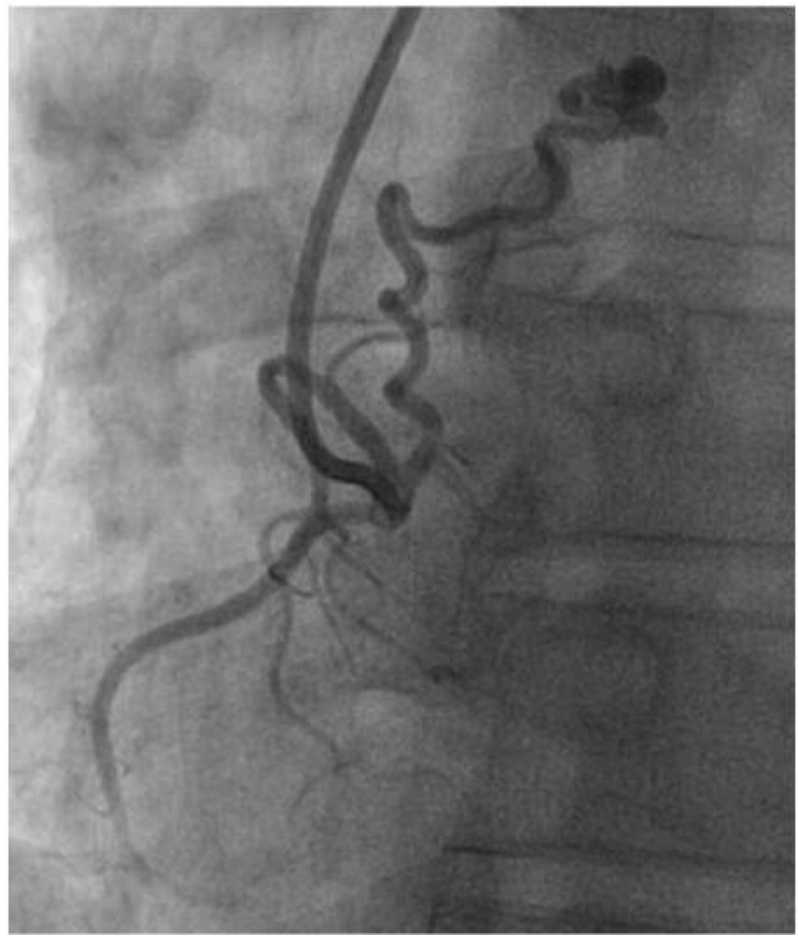

Fig. 1. A fistula originating from the proximal portion of RCA draining into the pulmonary artery. 


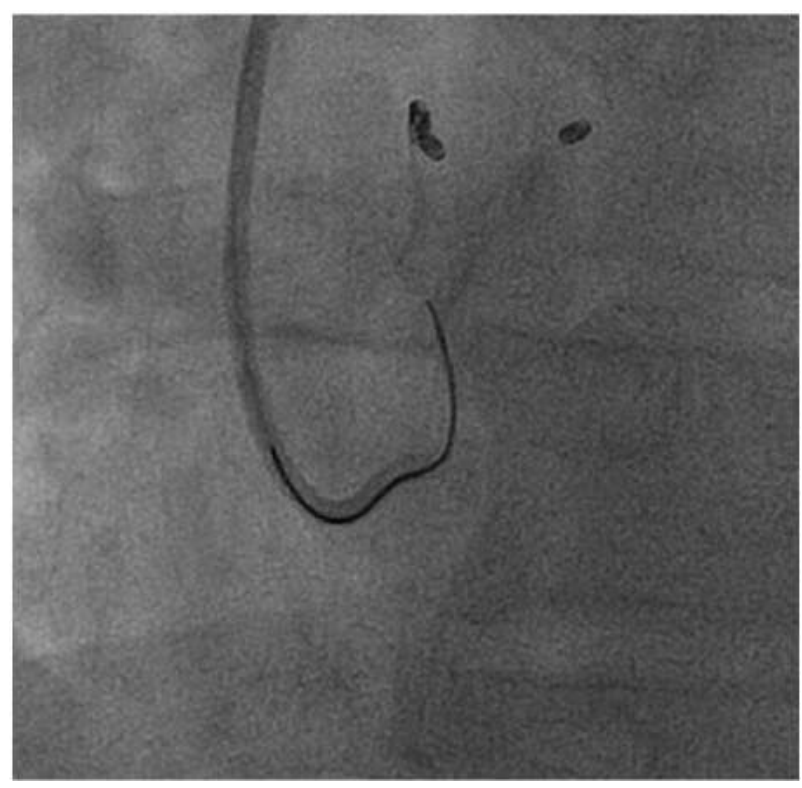

Fig. 2. Second coil placement.

patients and fistulas with a high flow rate. Percutaneous closure is the preferred technique, in particular in the absence of multiple fistulas and large fistula branches, and in cases where the fistula can be easily accessed. ${ }^{1,2}$ With catheter closure techniques, complete occlusion of the fistula may be achieved in $>95 \%$ of the patients. The main complications include either premature deflation of a detachable balloon, inadvertent coil embolisation, transient T-wave changes, transient bundle branch block and myocardial infarction. All the complications are rare, except for inadvertent coil migration, which may occur as a result of high flow in the large fistulas or with undersized coils. ${ }^{3,4}$ In our patient migration of the coil was due to fast contrast medium injection. Even if the coils do migrate, they can be pulled back with snares. In our

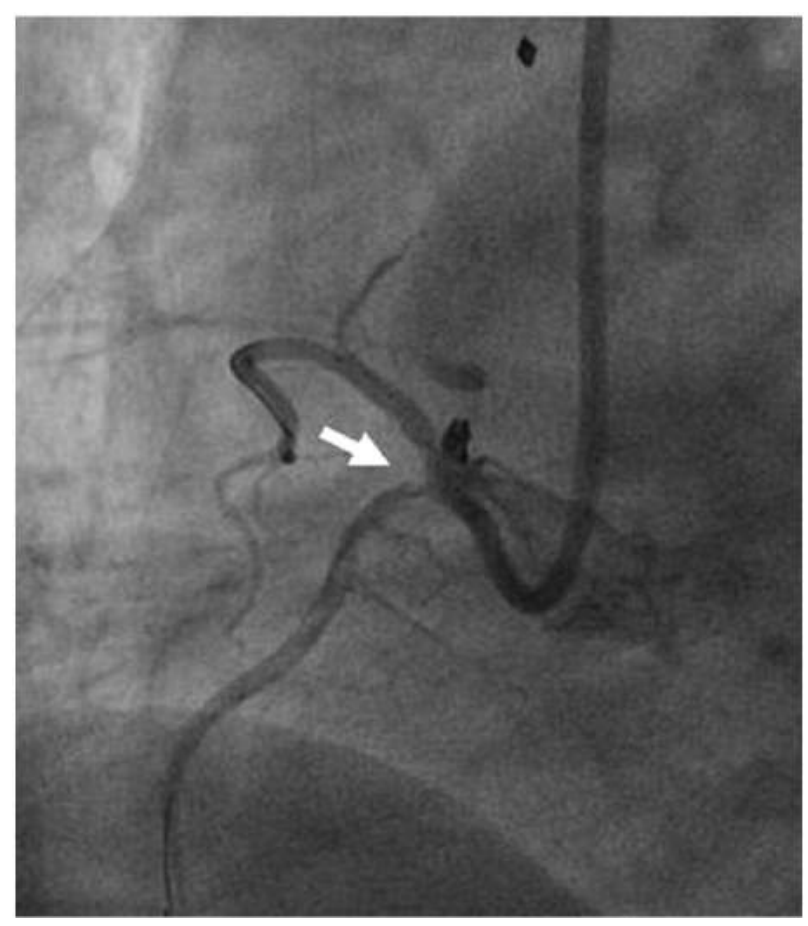

Fig. 3. Coil migration and coronary dissection in the osteal part of right coronary artery (arrow).

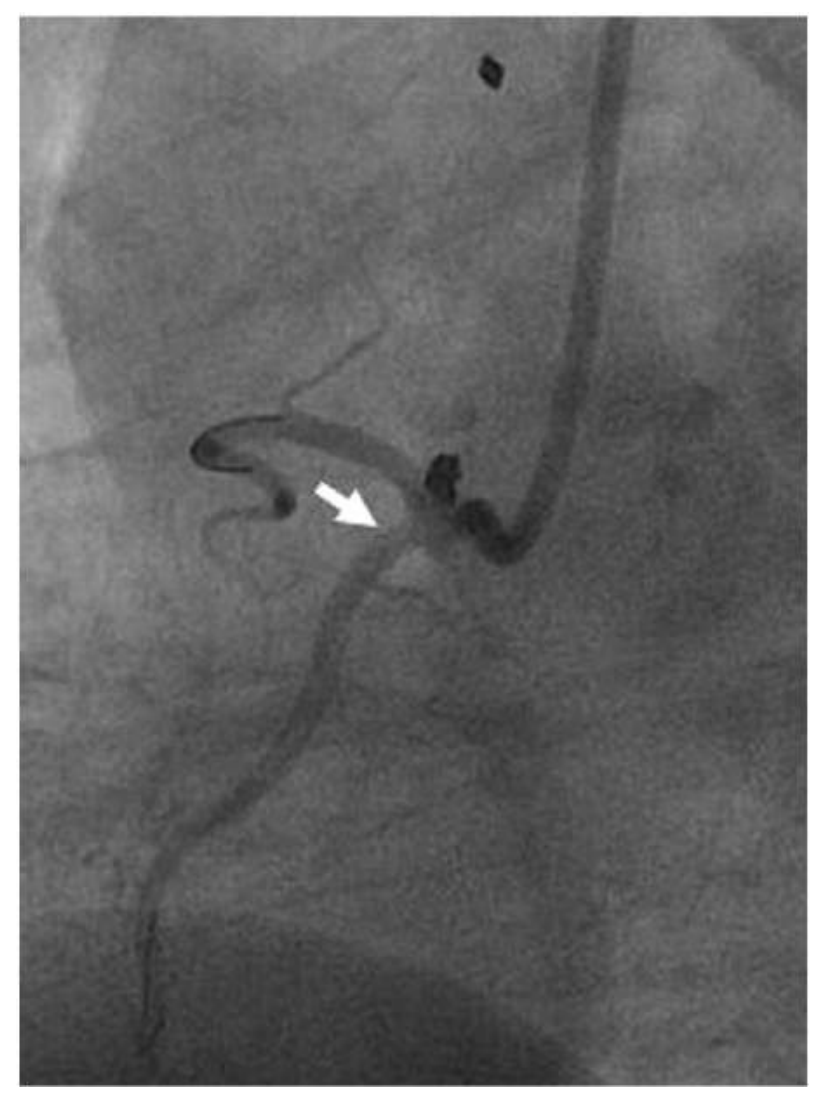

Fig. 4. Bare metal stent implantation to cover the dissected segment and to jail migrated coil (arrow).

knowledge this is the first case with coil migration and coronary dissection to occur simultaneously in the same patient.

This case shows the unusual complication of percutaneous closure of coronary artery fistulas and successful management of this complication using stent implantation.

\section{Conflict of interest}

None.

\section{References}

1. Gowda RM, Vasavada BC, Khan IA. Coronary artery fistulas: clinical and therapeutic considerations. Int J Cardiol 2006;107:7-10.

2. Jiang Z, Chen H, Wang J. Right coronary artery fistula to left ventricle treated by transcatheter coil embolization: a case report and literature review. Intern Med 2012;51: 1351-1353.

3. Reidy JF, Anjos RT, Qureshi SA, Baker EJ, Tynan MJ. Transcatheter embolization in the treatment of coronary artery fistulas. J Am Coll Cardiol 1991;18:187-192.

4. Qureshi SA, Reidy JF, Alwi MB, et al. Use of interlocking detachable coils in embolizationof coronary arteriovenous fistulas. Am J Cardiol 1996;78:110-113. 